\title{
Aceitação do Mobile Banking no Brasil: uma Análise Por Meio do Modelo TAM Estendido
}

\author{
Evandro Luiz Lopes \\ Universidade Nove de Julho - São Paulo - Brasil \\ Universidade Federal de São Paulo - São Paulo - Brasil \\ E-mail - elldijo@uol.com.br \\ orcid.org/0000-0002-2780-4215 \\ Laís Lima Caracciolo \\ Universidade Federal de São Paulo - São Paulo - Brasil \\ E-mail - caraciolo_lais@gmail.com \\ Eliane Herrero \\ Universidade Nove de Julho - São Paulo - Brasil \\ E-mail - liaherrero@gmail.com \\ orcid.org/oooo-0002-4515-7242
}

\begin{abstract}
Resumo
Não existem garantias de que os avanços tecnológicos serão amplamente absorvidos e postos em prática. Um influente modelo de aceitação de tecnologia, o TAM, postula que percepções individuais sobre novas tecnologias - Utilidade Percebida (UP) e Facilidade de Uso Percebida (FUP) - podem ser utilizadas para prever sua aceitação, expressa pela Intenção de Uso (IU). Em telecomunicações, o número de consumidores que utilizam tecnologias idênticas ou compatíveis é um fator que influencia o comportamento de novos consumidores. Combinando o modelo TAM e a proposição de que o número de usuários também influencia o processo de tomada de decisão do consumidor em determinado contexto, o objeto deste estudo foi examinar os efeitos da percepção do número de usuários e o valor percebido da nova tecnologia, como antecedente do modelo TAM, no processo de adoção e uso do Mobile Banking no Brasil. Assim, conduzimos um survey com 147 estudantes universitários, cujos dados foram analisados por meio da modelagem de equações estruturais. Os resultados comprovam a eficiência do modelo TAM para estimar a intenção de uso de novas tecnologias e também que o número de usuários percebido pelo adotante da nova tecnologia exerce influência positiva sobre sua intenção de uso.
\end{abstract}


Palavras-chave: aceitação tecnológica, intenção de uso, comportamento do consumidor, mobile banking 


\title{
The Acceptance of Mobile Banking in Brazil: an Analysis of the Model Extended TAM Model
}

\author{
Evandro Luiz Lopes \\ Universidade Nove de Julho - São Paulo - Brasil \\ Universidade Federal de São Paulo - São Paulo - Brasil \\ E-mail - elldijo@uol.com.br \\ orcid.org/0000-0002-2780-4215 \\ Laís Lima Caracciolo \\ Universidade Federal de São Paulo - São Paulo - Brasil \\ E-mail - caraciolo_lais@gmail.com \\ Eliane Herrero \\ Universidade Nove de Julho - São Paulo - Brasil \\ E-mail - liaherrero@gmail.com \\ orcid.org/oooo-0002-4515-7242
}

\begin{abstract}
There is no guarantee that technological advances will be widely absorbed and put into practice. One influential model of technology acceptance, TAM, postulates that individual perceptions regarding a new technology - Perceived Utility (UP) and Perceived Usability (FUP) - can be used to predict its acceptance, expressed by the Intention of Use (IU). In telecommunications, the number of consumers using identical or compatible technologies also becomes a factor that influences and interferes with the behavior of new consumers. Combining the TAM model and the proposition that the number of users also influences the decision-making process of the consumer in a given context, the object of this study was to examine the effects of the perception of the number of users and the perceived value of the new technology, such as TAM model, in the process of adoption and use of Mobile Banking in Brazil. Thus, we conducted a survey with 147 university students, whose data were analyzed through structural equations modeling. The results confirm the efficiency of the TAM model to estimate the intention to use new technologies, also that the number of users perceived by the adopters of the new technology exerts a positive influence on their intention to use.
\end{abstract}


Keywords: technological acceptance, intention of use, consumer behavior, mobile banking 


\section{Introdução}

Um dos fenômenos recentes desencadeados pela disseminação do acesso à web por meio das tecnologias móveis é o uso do Mobile Banking. O crescimento do número de usuários dessa nova tecnologia é impressionante.

Mesmo sob essa condição, poucos estudos buscaram entender os motivos que levam os usuários a adotar - ou intencionar adotar - essa nova ferramenta. Mesmo assim, várias instituições bancárias têm realizado altos investimentos no desenvolvimento e manutenção de suas plataformas de Mobile Banking. Os poucos estudos sobre o tema realizados no Brasil tiveram como escopo principal identificar os resultados da experiência de uso (Ramos, Pimenta, \& Rodrigues, 2010) ou buscaram conhecer as diferenças perceptuais entre adotantes e não adotantes da plataforma (Püschel, Mazzon, \& Hernandez, 2010).

Mesmo que a literatura já conte com diversos trabalhos que utilizaram o modelo de adoção de tecnologia proposto por Davis (1986) (veja por exemplo: Sánchez-Prieto, OlmosMigueláñez, \& García-Peñalvo, 2017, Chintalapati \& Daruri, 2017, Islam, \& Buxmann, 2018), acreditamos que o modelo é bastante adequado para explicar a intenção em adotar o Mobile Banking no cenário nacional. Essa crença é fundamentada na robustez do modelo (Rauniar, 2014; Chintalapati \& Daruri, 2017; Almasri, 2018), no poder psicométrico já identificado na ferramenta (Gangwar, Date, \& Ramaswamy, 2015; Mohammadi, 2015) e em sua validade preditiva que já foi amplamente comprovada ( $\mathrm{Ni}, \mathrm{Lu}, \& \mathrm{Liu}, 2018$; Rachman \& Napitupulu, 2018)

Em um estudo anterior, Wang, Fang e Lo (2008) propuseram duas novas variáveis antecedentes do modelo TAM. Os autores estimaram o valor da tecnologia percebida e a percepção do número de usuários da nova tecnologia como variáveis independentes do modelo de adoção de tecnologias. Os resultados foram promissores e as escalas utilizadas mostraram-se confiáveis e consistentes.

Diante desse cenário, elaboramos o seguinte problema de pesquisa: qual o efeito da percepção da quantidade de usuários e o valor percebido da nova tecnologia na intenção de uso do Mobile Banking no Brasil? Com isso, o objetivo principal deste estudo é verificar o efeito da percepção do número de usuários e o valor percebido da nova tecnologia, como antecedente do modelo TAM, na intenção de adoção do Mobile Banking por consumidores brasileiros. Para isso, utilizamos o modelo TAM, proposto por Davis (1986) e adaptado por Wang, Fang e Lo (2008). 
A relevância deste estudo dá-se tanto pela perspectiva teórica quanto pela gerencial. Teoricamente, há uma lacuna acerca de como a percepção do número de usuários pode influenciar não usuários na adoção de uma nova tecnologia. Também, ainda são parcos os estudos que analisaram o efeito da percepção do valor da nova tecnologia na intenção de adoção do consumidor. Do ponto de vista gerencial, a relevância é justificada pelo grande investimento que os bancos estão fazendo no desenvolvimento de suas plataformas mobile. Em alguns casos, o investimento nesta nova plataforma supera os investimentos realizados no desenvolvimento de outras soluções online, como os websites por exemplo (Zhou, 2018)

Para relatar os resultados do survey realizado, este artigo está estruturado em outras quatro seções, além desta breve introdução. Na segunda seção, apresentamos a revisão da literatura e as hipóteses que foram testadas. Na seção seguinte, explanamos o método utilizado na fase empírica do estudo. Na quarta seção, apresentamos os resultados obtidos e, na quinta, há as considerações finais sobre esta pesquisa.

\section{Revisão Teórica}

O objetivo deste capítulo é apresentar as principais bases teóricas que nortearam nosso estudo. Iniciamos a seção apresentando o contexto substantivo da nossa aplicação e, na sequência, apresentamos a revisão da literatura utilizada na construção das hipóteses deste estudo.

\section{O Mobile Banking No Brasil}

O crescimento do Mobile Banking no Brasil é notável. Desde 2009, percebe-se um aumento expressivo da utilização desse canal para efetivação das transações bancárias no país. Segundo o relatório anual sobre tecnologia bancária da Federação Brasileira de Bancos (FEBRABAN, 2013), a conveniência trazida pelo uso do Mobile Banking foi tão notável que não só fez com que clientes abandonassem visitas físicas a outros canais como também os tornou mais ativos no relacionamento transacional com os bancos, justificando parte do aumento dessas transações, quando comparadas com períodos anteriores.

O Mobile Banking (MB, deste ponto em diante) é caracterizado pelo uso de dispositivos móveis - smartphones e tablets - para acesso e efetivação de transações bancárias via web (Zarifopoulos \& Economides, 2008). 
Entre 2009 e 2013, o número de contas correntes habilitadas para uso do MB passou de 400 mil para 11,7 milhões em 2013, justificado pelo expressivo aumento de usuários de smartphones no mesmo período. Ressalta-se também que a maior parte das transações bancárias utilizadas por esse canal são as que não contemplam movimentações financeiras, ou seja, o cliente está, cada vez mais, exercendo um acompanhamento assíduo de sua conta-corrente, devido à grande facilidade que o canal lhe propõe. Contudo, mesmo registrando um crescimento exponencial, ainda há bastante espaço para crescimento e desenvolvimento da tecnologia no Brasil (Kupczik, 2009).

Em 2015, o MB obteve o melhor resultado de sua história - foi o primeiro ano em que assumiu a segunda posição entre os canais preferidos pelos brasileiros para realização de transações bancárias, contando com 33 milhões de contas ativas.

A consolidação dos canais digitais é o que está sendo vivido no momento, já que os consumidores buscam a virtualização, acessibilidade e facilidade de uso. Os anseios por expansão e investimentos no canal mobile estão mais aquecidos do que nunca, principalmente quando consideramos o potencial de crescimento e melhoria da acessibilidade à internet pela população. Cada vez mais, o número de usuários do MB tende a aumentar. Esse crescimento é justificado tanto pela aceleração do uso de smartphones no país quanto pelo investimento realizado pelos bancos para a expansão dos serviços prestados por esse canal (Kupczik, 2009; Santos, Veiga, \& Moura, 2011; Silva, Schroder, \& Kroenke, 2018).

\section{A Evolução Do Modelo De Aceitação Tecnológica}

Os modelos advindos da psicologia social são uma importante fonte teórica de pesquisas para investigação dos determinantes do comportamento de uso. A Teoria da Ação Racional (TAR) é um dos modelos de intenção mais bem desenvolvidos e estudados, se mostrando bem-sucedido no processo de previsão e explicação do comportamento do consumidor em vários campos e domínios. A TAR é uma teoria geral, concebida para explicar virtualmente qualquer comportamento humano.

Davis (1986), com base na TAR, introduziu o modelo TAM (Technology Acceptance Model).

De acordo com a TAR, a manifestação de um comportamento específico é governada pela intenção comportamental, a qual é determinada pela atitude que, por sua vez, é 
determinada pelas crenças do indivíduo. No modelo TAM, Davis (1986) aplica essa sequência conceitual e estende a teoria TAR original.

Davis sugere que a intenção comportamental determina o uso da tecnologia. A partir dessa premissa, o TAM utiliza a TAR como uma base teórica para explicar, especificamente, as relações causais entre a utilidade percebida e a facilidade de uso percebida e entre a atitude, a intenção do usuário e a adoção da tecnologia em si (Davis, 1986).

A proposta principal do TAM é prover uma base teórica para analisar o impacto de fatores externos nos princípios internos, atitudes e intenções dos consumidores, baseandose em um pequeno número de variáveis fundamentais sugeridas por outros estudos (Davis, 1986; Pando-Garcia, Periañez-Cañadillas, \& Charterina, 2016) e relacionadas com os determinantes racionais e afetivos da aceitação da tecnologia.

O modelo TAM enuncia dois fatores como sendo extremamente relevantes na aceitação de novas tecnologias: a) a Utilidade percebida (UP), que é definida como uma prospecção da probabilidade subjetiva de que o uso em um contexto específico irá aumentar a performance da atividade, e b) a Facilidade de Uso Percebida (FUP), que se refere à facilidade adquirida ou à diminuição de esforços na realização de alguma tarefa.

Sendo assim, é postulado o fato de que a adoção de novas tecnologias é definida da seguinte forma:

$$
\mathrm{IU}=\mathrm{A}+\mathrm{UP}
$$

Na equação, IU é a Intenção de Uso e “A” se refere à Atitude de Utilização. Dessa forma, a Intenção de Uso estaria sendo diretamente influenciada pela Atitude do uso em si e pela Utilidade Percebida.

De acordo com o modelo TAM, a Atitude orientada ao uso é designada pela Utilidade Percebida e pela Facilidade de Uso Percebida, com seus relativos pesos estatísticos, determinados pela regressão linear:

$$
\mathrm{A}=\mathrm{UP}+\mathrm{FUP}
$$

O fluxo do modelo TAM (Figura 1) foi projetado para compreender a relação causal entre variáveis externas de aceitação dos usuários e o uso real da tecnologia, buscando entender o comportamento desses usuários, por meio do conhecimento da utilidade e da facilidade de utilização percebida por eles (Davis, 1989). 


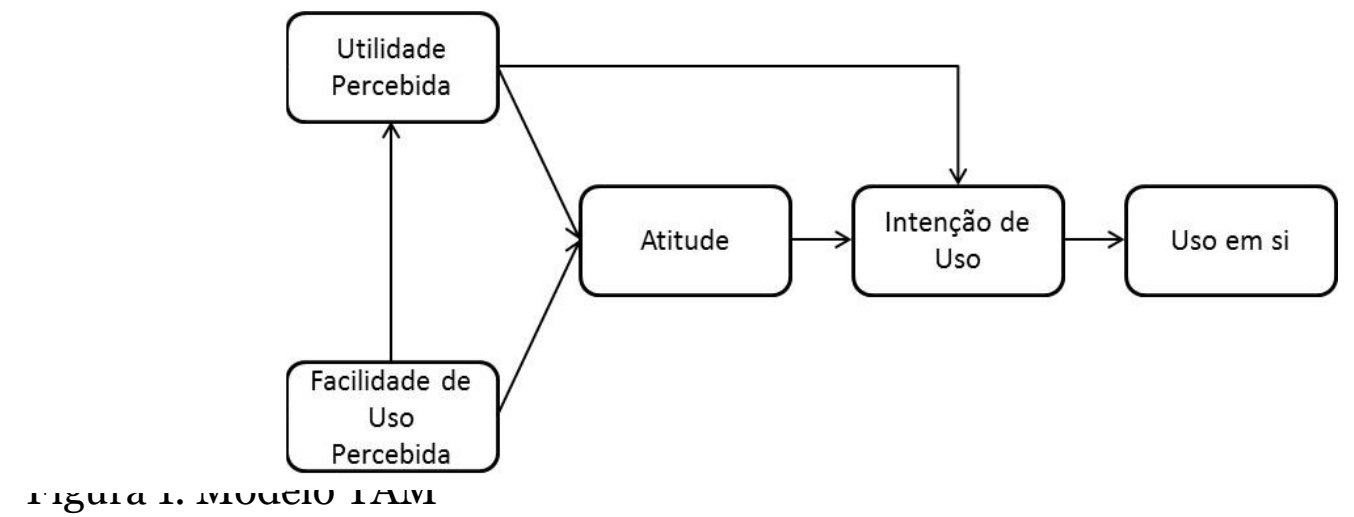

Fonte: Adaptado de Davis, Bagozzi, e Warshaw (1989) e Davis (1989).

Em resumo, de acordo com o modelo TAM, a Utilidade Percebida (UP) e a Facilidade de Uso Percebida (FUP) são os fatores que guiam a intenção de compra ou uso de um produto ou serviço.

As variáveis externas formam a intenção comportamental de uso (ou de não uso) da tecnologia, por meio do seu impacto entre utilidade percebida e facilidade de uso; por isso um dos principais efeitos do TAM é fornecer uma base para o rastreio dessas variáveis.

O TAM é o modelo mais relevante no processo de entendimento e previsão da aceitação tecnológica, pois considera que as percepções individuais, o comportamento do usuário e as intenções de utilizar um sistema são, de fato, determinados pela utilidade percebida e pela facilidade de uso percebida (Wang, Fang, \& Lo, 2008).

\section{TAM Estendido}

Desde a publicação do estudo de Davis (1989), outros pesquisadores buscaram refinar o modelo TAM, principalmente quanto às suas variáveis antecedentes.

\section{Externalidades de Rede}

O processo de aceitação de alguns produtos e serviços depende do número de usuários que adquiriram serviços compatíveis (Katz \& Shapiro, 1996). Esse fenômeno é chamado de externalidade de rede e é considerado, em uma abordagem econômica, fundamental na aceitação e difusão de um bem (Zott \& Amit, 2010). 
A externalidade de rede nada mais é do que a soma dos efeitos causados em um usuário, decorrentes da percepção de que outros usuários utilizam aquele item (Hong, Cao, \& Wang, 2017). Também chamada de “efeito em rede”, essa característica vem sendo definida como uma alteração no benefício percebido em um produto ou serviço, quando o número de agentes que consome o mesmo se altera (Torrent-Sellens, 2015).

Quando um produto é caracterizado pela externalidade de rede, o número de usuários ou a base inicial de adeptos determina significativamente a aceitação do consumidor. Uma tecnologia com uma base inicial de usuários menor é mais suscetível a experimentar uma barreira na aceitação, enquanto aquela com mais adeptos tende a ser mais efetiva (Anderson Jr., Parker, \& Tan, 2013; Hong, Cao, \& Wang, 2017).

Produtos com externalidades de rede exibem características diferentes de produtos que não as possuem. Usuários de bens que não são afetados pelo efeito rede consideram o principal benefício da inovação tecnológica antes de adotar a tecnologia, enquanto a aceitação de uma tecnologia, na presença da externalidade, não é determinada apenas pelo valor da tecnologia, mas também pelo valor relativo da rede (Farrel \& Saloner, 1996).

Muitos produtos são associados com externalidades de rede e vêm sendo analisados sob esse aspecto. Exemplos famosos incluem o uso do ATM (Automated Teller Machine), na indústria bancária (Hannan \& Mcdowell, 1984), contas eletrônicas e sistema de pagamento online (Kauffman, 2001), ferramentas de planilhas online e programação (Gandal, 1994) e sistemas informacionais (Conner, 1995; Chismar \& Meier,1992).

Entre todos os produtos relacionados ao efeito de rede, os primeiros exemplos reconhecidos surgiram no contexto das telecomunicações (Battistella, Colucci, De Toni, \& Nonino, 2013). É nesse setor, mais expressivamente das telecomunicações móveis, que podemos observar com clareza que o valor de um serviço para um usuário aumenta de acordo com o número de adotantes, e assim o serviço se torna cada vez mais atrativo para os não usuários (Battistella et al., 2013; Masson, Jain, Ganesh, \& George, 2016).

Wang, Fang e Lo (2008) agregam ao processo decisório desenhado pelo TAM duas novas variáveis relevantes: a compatibilidade e o valor da tecnologia propriamente dito. $\mathrm{O}$ valor da tecnologia é o benefício independente, derivado da própria, chamado pela literatura de "benefício único", "valor específico da tecnologia” ou ainda "TSV (Tecnology Specific Value)”. Já a compatibilidade é o benefício derivado do efeito de rede e transcreve a tendência de consumidores que consideram o valor adicionado por outros usuários que possuem produtos similares ou complementares (Kauffman, McAndrews, \& Wang, 2000). 
O efeito de rede, na maioria dos casos, é determinado por dois fatores, sendo o número de outros usuários que usam produtos compatíveis e o número de bens complementares (Wang, Fang, \& Lo, 2008). Os efeitos derivados de produtos complementares nem sempre estão presentes nas tecnologias propostas pelo setor das telecomunicações (Chih-Chien, Hsu, \& Fang, 2005; Masson et al., 2016). Sendo assim, uma vez que o número de bens complementares não é existente, o benefício das externalidades de rede é determinado apenas pelo número dos usuários (Chih-Chien, Hsu, \& Fang, 2005; Wang, Fang, \& Lo, 2008).

\section{O Modelo TAM Estendido às Telecomunicações Móveis}

Originalmente, a relação trazida pelo TAM descreve que a intenção de uso é dada por dois construtos - a utilidade percebida (UP) e a facilidade de uso percebida (FUP). Wang, Fang e Lo (2008) relacionam o valor da tecnologia e o número de usuários em um modelo estendido (veja a Figura 2). Tal modelo segue os estudos conduzidos por Venkatesh e Davis (2000) e adiciona construtos, relacionados às externalidades de rede.

$\mathrm{O}$ atual comportamento de uso e adoção de uma nova tecnologia na área de telecomunicações móveis é determinado pela intenção de uso do consumidor. Essa intenção, em relação à tecnologia, é afetada diretamente, e ao mesmo tempo, pela utilidade percebida e pela facilidade de uso percebida. Esta última também influencia, indiretamente, a intenção de uso, uma vez que influencia a utilidade percebida.

O valor da tecnologia e o número de usuários são adicionados ao TAM original e cria-se a hipótese de que essas variáveis afetam positivamente a utilidade percebida. Esse TAM estendido pode ser usado para mensurar a aceitação de inovações na área de telecomunicações móveis e de qualquer outra tecnologia que possua externalidades de rede.

O modelo de Wang, Fang e Lo (2008) foi testado em Taiwan, com o objetivo de prever o comportamento de adoção a inovações na área de telecomunicações móveis, por meio de uma pesquisa empírica que buscou avaliar a aceitação do serviço de MMS (Multimedia Messaging Service), uma tecnologia nova à época e que compartilhava os efeitos de rede.

O resultado obtido suporta a tese e a acuracidade do modelo estendido. Os consumidores consideraram o número de usuários do serviço tão relevante quanto o 
benefício agregado puramente pela nova tecnologia, quando estavam tomando sua decisão sobre adotar ou não o serviço (Wang, Fang, \& Lo, 2008).

Todos os caminhos postulados pelo modelo estendido se mostraram significantes ( $\mathrm{p}<0,05)$. Como indicado na Figura 2, tanto o TSV quanto o número de usuários influenciam positivamente a UP e afetam, indiretamente, a intenção de uso por meio da UP, o que implica que o TAM e as externalidades de rede são integrados ao modelo estendido de forma bem-sucedida.

Nesse novo modelo, a UP e a FUP também determinam diretamente a intenção de uso. A FUP, indiretamente, também determina a intenção de uso por meio da UP. Ou seja, o conceito TAM original foi completamente incorporado ao novo modelo.

\section{Formulação Das Hipóteses}

A fim de verificar a relevância do valor da tecnologia e do número de usuários no processo de aceitação do MB no Brasil, são propostas as seguintes hipóteses, baseadas no modelo formulado por Wang, Fang e Lo (2008):

H1 - Existe relação positiva entre o valor da tecnologia e a utilidade percebida do uso do MB.

H2 - existe relação positiva entre a percepção do número de usuários e a utilidade percebida do uso do MB.

A essas proposições, foram incorporadas as hipóteses postuladas por Davis e Venkatesh (2000), para assim concebermos e testarmos um modelo integrativo dos construtos que englobam todas as variáveis significantes do fenômeno estudado. Sendo assim, propomos:

H3 - Existe relação positiva entre utilidade percebida e intenção de uso do uso do MB.

H4a - Existe relação positiva entre a facilidade de uso percebida e a utilidade percebida do uso do MB.

$\mathrm{H} 4 \mathrm{~b}$ - Existe relação positiva entre a facilidade de uso percebida e a intenção de uso do MB.

Finalizando essa seção, a Figura 2 apresenta o modelo teórico proposto, com a indicação das hipóteses que foram testadas na fase empírica deste trabalho. 


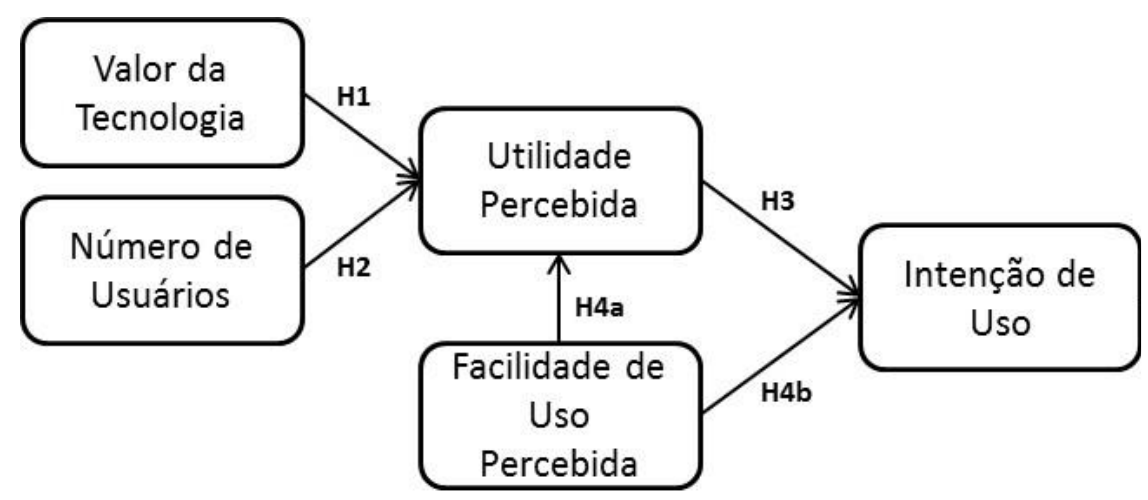

Figura 2. Modelo conceitual proposto

Fonte: adaptado de Davis (1989) e de Wang, Fang e Lo (2008)

\section{Método}

Dada a necessidade de adaptação do modelo TAM estendido para o serviço de MB no mercado brasileiro, o estudo empírico foi realizado por meio de um survey quantitativo descritivo, feito em corte transversal (Malhotra, 2012).

Assumiu-se que a comunidade universitária brasileira seria o público adequado para aplicação do survey, uma vez que são os jovens o grupo de indivíduos com maior propensão a estarem imersos nos produtos e serviços relacionados ao setor de telecomunicações (Wang, Fang, \& Lo, 2008). Ademais, mesmo que o uso de estudantes universitários resulte em amostras mais homogêneas (Peterson, 2001), estudos já identificaram que essa amostra é adequada para pesquisas do comportamento do consumidor (Goodman, Cryder, \& Cheema, 2013; Peterson \& Merunka, 2014; Bhatti, 2015, entre outros).

\section{Instrumento De Coleta De Dados}

Utilizamos um formulário em que foram aplicadas escalas para mensuração dos construtos que formaram o modelo teórico de pesquisa (valor da tecnologia, percepção do número de usuários, utilidade percebida, facilidade de uso percebida e intenção de uso). 
Para esta mensuração, foram utilizadas escalas adaptadas de trabalhos anteriores, como descrito na Tabela 1.

Tabela 1

Escalas utilizadas

\begin{tabular}{|c|c|c|c|}
\hline Construto & Rótulos & Afirmativa & Fonte \\
\hline \multirow{3}{*}{$\begin{array}{l}\text { Valor da } \\
\text { tecnologia }\end{array}$} & Val_tec1 & O Mobile Banking é uma tecnologia útil. & \multirow{7}{*}{$\begin{array}{c}\text { Adaptado } \\
\text { de Wang, } \\
\text { Fang, e Lo } \\
(2008)\end{array}$} \\
\hline & Val_tec2 & O Mobile Banking é uma grande inovação tecnológica. & \\
\hline & Val_tec3 & $\begin{array}{l}\text { O Mobile Banking é um serviço que agregou grande valor às } \\
\text { telecomunicações. }\end{array}$ & \\
\hline \multirow{4}{*}{$\begin{array}{l}\text { Percepção do } \\
\text { número de } \\
\text { usuários }\end{array}$} & Perc_usu1 & $\begin{array}{l}\text { Na minha opinião, o número de usuários do MB em smartphones é } \\
\text { grande. }\end{array}$ & \\
\hline & Perc_usu2 & $\begin{array}{l}\text { Muitas pessoas com quem me relaciono usam o serviço de internet } \\
\text { banking no celular. }\end{array}$ & \\
\hline & Perc_usu3 & $\begin{array}{l}\text { A maioria dos celulares utilizados estão preparados para receber a } \\
\text { tecnologia de internet banking. }\end{array}$ & \\
\hline & Perc_usu4 & $\begin{array}{l}\text { Muitos usuários de smartphone utilizam o serviço de internet } \\
\text { banking no celular. }\end{array}$ & \\
\hline \multirow{4}{*}{$\begin{array}{c}\text { Facilidade de } \\
\text { uso } \\
\text { Percebida }\end{array}$} & Fac_uso1 & Considero a utilização do Mobile Banking clara e objetiva. & \multirow{10}{*}{$\begin{array}{c}\text { Adaptado } \\
\text { de Davis e } \\
\text { Venkatesh } \\
(2000)\end{array}$} \\
\hline & Fac_uso2 & Interagir com essa tecnologia não requer muito esforço mental. & \\
\hline & Fac_uso3 & Considero a tecnologia fácil de ser utilizada. & \\
\hline & Fac_uso4 & $\begin{array}{l}\text { Considero fácil fazer com que a tecnologia responda aos meus } \\
\text { requerimentos. }\end{array}$ & \\
\hline \multirow{4}{*}{$\begin{array}{l}\text { Utilidade } \\
\text { Percebida }\end{array}$} & Uti_perc1 & $\begin{array}{l}\text { A utilização dessa tecnologia melhorou minha performance no dia- } \\
\text { a-dia. }\end{array}$ & \\
\hline & Uti_perc2 & $\begin{array}{l}\text { A utilização dessa tecnologia no meu dia-a-dia aumentou minha } \\
\text { produtividade. }\end{array}$ & \\
\hline & Uti_perc3 & A utilização dessa tecnologia me tornou mais eficiente. & \\
\hline & Uti_perc4 & Considero a tecnologia útil ao meu dia-a-dia. & \\
\hline \multirow{2}{*}{$\begin{array}{l}\text { Intenção de } \\
\quad \text { uso }\end{array}$} & Int_uso1 & Assumindo que eu tenho acesso ao Mobile Banking, eu utilizaria. & \\
\hline & Int_uso2 & $\begin{array}{l}\text { Caso eu tivesse acesso ao Mobile Banking, eu teria intenção de } \\
\text { utilizá-lo. }\end{array}$ & \\
\hline
\end{tabular}

Mesmo que o construto Intenção de uso tenha sido operacionalizado apenas com dois indicadores e isso possa ser entendido como um limite (Churchill \& Iacobucci, 2006), optamos por manter a escala original como forma de manter a integridade psicométrica do modelo (Churchill \& Iacobucci, 2006, Furr, 2017).

Todos os itens foram estimados por meio de uma escala do tipo Likert de 7 pontos, ancorados entre 1 (discordo totalmente) e 7 (concordo totalmente).

\section{Amostra e Procedimentos de Campo}


Antes da aplicação do questionário, conduzimos um pré-teste, com a apresentação do formulário de coleta de dados para cinco indivíduos (três homens e duas mulheres) com o mesmo perfil da amostra. Esse pré-teste não resultou em nenhuma alteração significativa do instrumento. Os questionários preenchidos nessa fase foram descartados do estudo.

Para o cálculo do tamanho da amostra, utilizamos o software G*Power 3.0 (Faul, Erdfelder, Buchner, \& Lang, 2009). Os parâmetros utilizados foram: cinco preditores, effect size $\left(\mathrm{f}^{2}\right)$ de 0,15 e confiança de $95 \%(\alpha=0,05)$. Como apresentado na Figura 3 , a amostra adequada indicada pelo software foi de 138 indivíduos $(\mathrm{n}=138)$.

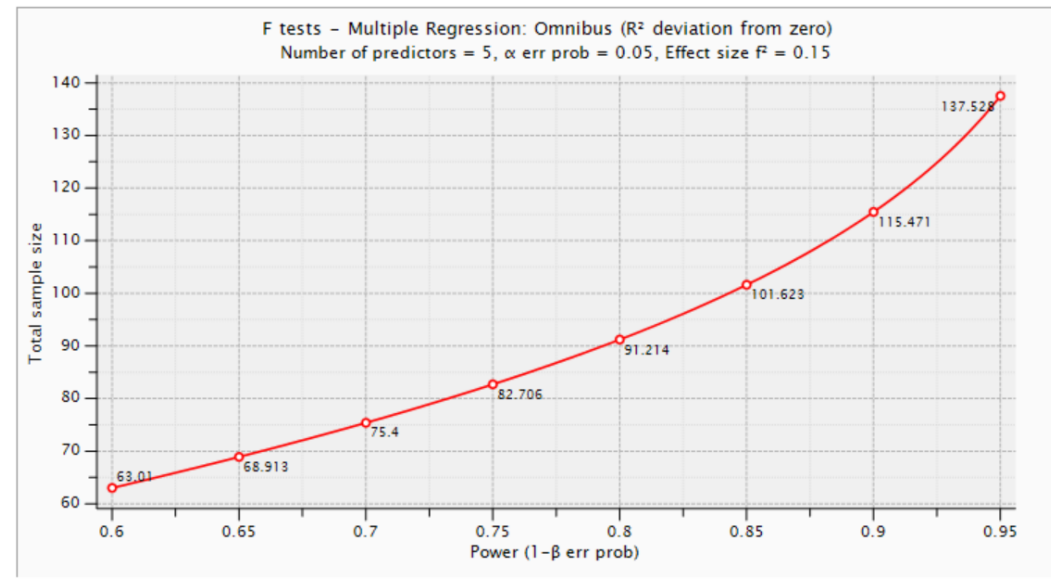

Figura 3. Definição do tamanho da amostra

Fonte: os autores com o uso do software $\mathrm{G}^{*}$ Power 3.0

O survey foi conduzido por meio da aplicação do instrumento de coleta de dados na própria sala de aulas. Os participantes foram convidados a preencher o instrumento de coleta de dados antes do início das aulas. Evitou-se a aplicação do instrumento em dias nos quais os estudantes realizariam provas ou outras atividades avaliativas (como apresentação de trabalhos e seminários, por exemplo). Explicamos que a atividade era voluntária e que o estudante decidiria se participaria ou não do estudo. Após as explicações gerais, os instrumentos de coleta de dados foram distribuídos e cada discente levou o tempo que achou necessário para o seu preenchimento.

\section{Plano De Análise Dos Dados.}

As análises iniciais do banco de dados gerado a partir do survey foram feitas com o uso do SPSS versão 18. Nessa fase, verificamos os pressupostos para a estimação das 
regressões que seriam realizadas. Conduzimos, portanto, a verificação da ausência de outliers, a análise da homocedasticidade, da multicolinearidade e da normalidade da distribuição dos dados.

A identificação de possíveis outliers univariados foi realizada por meio da análise dos gráficos box-plot, ao passo que a análise dos outliers multivariados foi conduzida por meio da estimação da distância de Mahalanobis (Churchill \& Iacobucci, 2006). A verificação da homocedasticidade foi realizada pela análise do teste de Levene e a verificação da ausência da multicolinearidade foi feita pelo cálculo dos fatores de inflação da variância (VIF). Finalmente, a verificação da normalidade foi conduzida por meio do cálculo do teste KS de Kolmogorov-Smirnov (Hair, Anderson, Tatham, \& Black, 2005).

Para análise dos relacionamentos entre os construtos, escolheu-se a técnica de modelagem de equações estruturais (MEE), por meio da matriz de correlação (Chin \& Newsted, 1999) e pelo método de estimação de mínimos quadrados parciais (PLS-PM), calculado com auxílio do software estatístico SmartPLS 2.o M3 (Ringle, Silva, \& Bido, 2014).

\section{Resultados Observados}

O objetivo geral deste capítulo é apresentar os principais resultados obtidos.

\section{Amostra}

O instrumento de coleta de dados foi distribuído para 400 estudantes universitários, porém $253(63,2 \%)$ foram descartados, pois os respondentes não possuíam contas bancárias. Do total válido, foram entrevistados 147 estudantes universitários de uma instituição de ensino superior pública brasileira, sendo 82 mulheres $(55,8 \%)$ e 65 homens (44,2\%). Os respondentes declararam idades entre 18 e 29 anos $(\mu=21 ; \sigma=2,3)$.

A maior parte dos respondentes afirmou utilizar os serviços de $\mathrm{MB}(\mathrm{n}=112 ; 76,2 \%)$ com boa frequência (ao menos quatro vezes por semana; $n=99 ; 67,4 \%$ ).

\section{Análises Iniciais do Banco de Dados}


Conduzimos as análises iniciais dos dados gerados no survey, observando os dados discrepantes. Contudo, nem os gráficos de blox-plot nem a distância de Mahalanobis (D²) identificaram outliers uni ou multivariados.

Identificamos ausência de heterocedasticidade, expressa por meio de indicadores de Levene não significantes ao nível de 10\% (Hair et al., 2005).

Também não foram observados problemas quanto à multicolinearidade dos dados, pois todos os indicadores de inflação da variância foram aceitáveis. Mesmo que não exista um limite crítico para essa comprovação (Hair et al., 2005; Churchill, \& Iacobucci, 2006), entende-se que VIFs inferiores a 10 indicam colinearidade aceitável.

Finalmente, não se observou a normalidade na distribuição dos dados, pois os indicadores KS foram não significantes ao nível de 5\%. Esse último achado reforça a decisão pela utilização da MEE pela estimação dos mínimos quadrados parciais, pois esta técnica não prescinde da aderência dos dados da curva gaussiana (Ringle, Silva, \& Bido, 2014).

Os resultados dessa fase são apresentados na Tabela 2.

Tabela 2

Análise inicial do banco de dados

\begin{tabular}{|c|c|c|c|c|c|c|c|c|c|c|c|c|}
\hline \multicolumn{6}{|c|}{ Estatísticas descritivas } & \multicolumn{4}{|c|}{ Homocedasticidade } & \multirow{2}{*}{\begin{tabular}{|c|} 
Colinearidade \\
VIF
\end{tabular}} & \multicolumn{2}{|c|}{ Normalidade } \\
\hline Variável & $\mathrm{n}$ & Mínimo & Máximo & $\mu$ & $\sigma$ & Levene & df1 & $\mathrm{df} 2$ & $\begin{array}{c}p- \\
\text { value }\end{array}$ & & $\mathrm{KS}$ & $\begin{array}{c}p- \\
\text { value }\end{array}$ \\
\hline Val_tec1 & 147 & 1 & 7 & 5,80 & 1,193 & ,063 & 1 & 145 & ,802 & 3,543 & 2,679 & ,000 \\
\hline Val_tec2 & 147 & 1 & 7 & 5,65 & 1,220 & ,034 & 1 & 145 &, 855 & 4,781 & 2,471 & ,000 \\
\hline Val_tec3 & 147 & 2 & 7 & 5,86 & 1,085 & ,897 & 1 & 145 &, 345 & 1,102 & 2,573 & ,000 \\
\hline Perc_usu1 & 147 & 2 & 7 & 5,90 & 1,096 & ,615 & 1 & 145 & ,434 & 5,594 & 2,628 & ,000 \\
\hline Perc_usu2 & 147 & 1 & 7 & 5,82 & 1,186 & ,296 & 1 & 145 &, 587 & 3,842 & 2,657 & ,000 \\
\hline Perc_usu3 & 147 & 1 & 7 & 4,68 & 1,490 &, 385 & 1 & 145 &, 536 & 8,870 & 2,011 & ,001 \\
\hline Perc_usu4 & 147 & 2 & 7 & 5,63 & 1,189 & ,040 & 1 & 145 & ,841 & 7,051 & 2,446 & ,000 \\
\hline Facil_Uso1 & 147 & 1 & 7 & 5,39 & 1,316 & ,215 & 1 & 145 & ,643 & 1,113 & 2,044 & ,000 \\
\hline Facil_Uso2 & 147 & 1 & 7 & 5,20 & 1,388 & ,291 & 1 & 145 & ,590 & 2,580 & 1,716 & ,006 \\
\hline Facil_Uso3 & 147 & 1 & 7 & 5,21 & 1,356 & ,466 & 1 & 145 & ,496 & 2,147 & 1,863 & ,002 \\
\hline Facil_Uso4 & 147 & 1 & 7 & 5,27 & 1,300 & ,031 & 1 & 145 & ,860 & 1,604 & 1,929 & ,001 \\
\hline Util_perc1 & 147 & 2 & 7 & 5,54 & 1,267 &, 048 & 1 & 145 & ,826 & 4,095 & 2,192 & ,000 \\
\hline Util_perc2 & 147 & 2 & 7 & 5,40 & 1,248 & ,047 & 1 & 145 & 829 & 5,556 & 2,111 & ,000 \\
\hline Util_perc3 & 147 & 3 & 7 & 5,56 & 1,165 & ,188 & 1 & 145 &, 665 & 2,841 & 2,220 & ,000 \\
\hline Util_perc4 & 147 & 3 & 7 & 5,71 & 1,154 & ,010 & 1 & 145 & ,921 & 3,670 & 2,327 & ,000 \\
\hline Int_Uso1 & 147 & 2 & 7 & 5,82 & 1,291 & ,160 & 1 & 145 & ,690 & 4,327 & 3,011 & ,000 \\
\hline Int_Uso2 & 147 & 2 & 7 & 5,81 & 1,273 & ,390 & 1 & 145 &, 533 & 4,520 & 2,907 & ,000 \\
\hline
\end{tabular}

Fonte: dados da pesquisa (2018) 
Finalizada essa fase inicial, passamos às análises das validades convergente e discriminante do modelo estrutural.

\section{Validade Convergente}

A validade convergente é uma medida da relação entre duas medidas do mesmo construto e indica a extensão pela qual a escala se relaciona positivamente. Um alto grau de validade convergente prova que duas medidas do mesmo construto estão relacionadas e, portanto, são conceitualizações adequadas (Bagozzi \& Phillips, 1982).

Para Chin (1998), a validade convergente é obtida quando se verificam cargas superiores a 0,50 entre os itens e os construtos. Todavia, outros autores sugerem medidas mais robustas, no sentido de se estabelecer a validade convergente, quando propõem que se eliminem os itens com carga inferior a 0,70 (Ringle, Silva, \& Bido, 2014).

$\mathrm{Na}$ presente aplicação, todos os itens apresentaram cargas superiores a 0,70 , atendendo aos pressupostos mais exigentes de validação. As cargas estão apresentadas na Figura 4 .

\section{Validade Discriminante}

Enquanto a validade convergente analisa o grau de correlação entre as variáveis, a validade discriminante representa o grau em que essas medidas se diferenciam entre si (Bagozzi \& Phillips, 1982). Ou seja, a validade discriminante avalia até que ponto uma variável latente não se correlaciona com outras, das quais se supõe que ela se diferencie (Ringle, Silva, \& Bido, 2014).

Em outras palavras, a variável discriminante é empregada para analisar o grau de distinção entre as variáveis que possuem medidas semelhantes, mas conceitos diferentes. Caso haja validade discriminante, as variáveis do construto terão maior poder de explicação para este do que para outro do modelo (Chin, 1998). Opostamente à validade convergente, a validade discriminante pressupõe que devam existir baixos índices de correlação entre as variáveis.

Na presenta análise, utilizamos o critério de Fornell e Larcker (1982), que postula que a validade discriminante é comprovada quando a raiz quadrada da variância média 
extraída do construto (AVE) é superior à correlação deste com os demais (Fornell \& Larcker, 1982; Chin, 1998).

A maior parte das raízes quadradas das AVEs apresentaram scores maiores que a correlação entre os demais construtos, como demonstrado na Tabela 3.

Tabela 3

Validade discriminante

\begin{tabular}{lcccccccc}
\hline \multicolumn{1}{c}{ Construto } & AVE & $\begin{array}{c}\text { Raiz } \\
\text { Quadrada } \\
\text { da AVE }\end{array}$ & 1 & 2 & 3 & 4 & 5 \\
\hline \hline $\begin{array}{l}\text { (1) Facilidade de uso } \\
\text { percebida } \\
\text { (2) Intenção de uso }\end{array}$ & 0,980 & 0,990 & 0,990 & & & & \\
$\begin{array}{l}\text { (3) Percepção do número de } \\
\text { usuários }\end{array}$ & 0,996 & 0,998 & 0,931 & 0,998 & & & \\
$\begin{array}{l}\text { (4) Utilidade Percebida } \\
\text { (5) Valor da tecnologia }\end{array}$ & 0,966 & 0,983 & 0,968 & 0,937 & 0,925 & 0,966 & \\
\hline
\end{tabular}

Nota: a diagonal em destaque indica a raiz quadrada da AVE do construto.

Fonte: dados da pesquisa (2018)

Porém, dada a alta correlação entre a Percepção do número de usuários com a Facilidade de uso percebida e a Utilidade percebida com a Facilidade de uso percebida, analisamos também a matriz de das cargas cruzadas entre os construtos (Ringle, Silva, \& Bido, 2014). A matriz é apresentada na Tabela 4.

Tabela 4

Matriz das cargas cruzadas

\begin{tabular}{lccccc}
\hline \multicolumn{1}{c}{$\begin{array}{c}\text { Variável } \\
\text { manifesta }\end{array}$} & $\begin{array}{c}\text { Facilidade de } \\
\text { uso percebida }\end{array}$ & Intenção de uso & $\begin{array}{c}\text { Percepção do número } \\
\text { de usuários }\end{array}$ & $\begin{array}{c}\text { Utilidade } \\
\text { percebida }\end{array}$ & $\begin{array}{c}\text { Valor da } \\
\text { Tecnologia }\end{array}$ \\
\hline \hline Facil_Uso1 & 0,9815 & 0,9191 & 0,9595 & 0,9728 & 0,9456 \\
Facil_Uso2 & 0,9929 & 0,9269 & 0,9556 & 0,9551 & 0,9462 \\
Facil_Uso3 & 0,9935 & 0,9186 & 0,9560 & 0,9516 & 0,9441 \\
Facil_Uso4 & 0,9927 & 0,9232 & 0,9531 & 0,9551 & 0,9370 \\
Int_Uso1 & 0,9272 & 0,9979 & 0,9546 & 0,9323 & 0,9539 \\
Int_Uso2 & 0,9312 & 0,9980 & 0,9617 & 0,9369 & 0,9622 \\
Perc_usu1 & 0,9405 & 0,9680 & 0,9770 & 0,9382 & 0,9750 \\
Perc_usu2 & 0,9406 & 0,9601 & 0,9738 & 0,9383 & 0,9852 \\
Perc_usu3 & 0,9106 & 0,8674 & 0,9418 & 0,9176 & 0,9042 \\
Perc_usu4 & 0,9406 & 0,9157 & 0,9751 & 0,9727 & 0,9617
\end{tabular}




\begin{tabular}{llllll} 
Util_perc1 & 0,9515 & 0,9259 & 0,9642 & 0,9890 & 0,9506 \\
Util_perc2 & 0,9729 & 0,9066 & 0,9496 & 0,9770 & 0,9313 \\
Util_perc3 & 0,9462 & 0,9087 & 0,9549 & 0,9877 & 0,9404 \\
Util_perc4 & 0,9357 & 0,9398 & 0,9653 & 0,9771 & 0,9693 \\
Val_tec1 & 0,9386 & 0,9600 & 0,9773 & 0,9435 & 0,9926 \\
Val_tec2 & 0,9417 & 0,9308 & 0,9749 & 0,9686 & 0,9806 \\
Val_tec3 & 0,9408 & 0,9526 & 0,9797 & 0,9441 & 0,9882 \\
\hline
\end{tabular}

Nota: as células destacadas apresentam as maiores cargas de cada item.

Fonte: dados da pesquisa (2018)

Como apresentado na Tabela 4, as maiores cargas fatoriais dos indicadores confirmaram a validade discriminante do modelo.

\section{Indicadores De Ajuste Do Modelo}

Além de verificarmos as validades convergente e discriminante, buscamos também identificar outros índices de ajustamento (Análise da variância extraída (AVE), Confiabilidade composta, Coeficiente de determinação ( $\left.{ }^{2}\right)$, Alfa de Cronbach, Comunalidade e Redundância). Esses indicadores estão apresentados na Tabela 5 .

\section{Tabela 5}

Indicadores gerais do modelo

\begin{tabular}{|c|c|c|c|c|c|c|}
\hline Construto & AVE & $\begin{array}{l}\text { Confiabilidade } \\
\text { composta }\end{array}$ & $\mathrm{R}^{2}$ & $\begin{array}{c}\text { Alfa de } \\
\text { Cronbach }\end{array}$ & Comunalidade & Redundância \\
\hline Facilidade de uso percebida & 0,980 & 0,995 & & 0,993 & 0,980 & \\
\hline Intenção de uso & 0,996 & 0,998 & 0,887 & 0,996 & 0,996 & 0,571 \\
\hline Percepção do número de usuários & 0,934 & 0,983 & & 0,976 & 0,934 & \\
\hline Utilidade Percebida & 0,966 & 0,991 & 0,962 & 0,988 & 0,966 & 0,584 \\
\hline Valor da tecnologia & 0,974 & 0,991 & & 0,987 & 0,974 & \\
\hline
\end{tabular}

Nota: os limites críticos segundo Hair et al. (2005) são: AVE >0,5; Confiabilidade Composta $>0,5$; Alfa de Cronbach >0,7; Comunalidade $>0,5$ e Redundância $<0,7$ Fonte: dados da pesquisa (2018)

Como pode ser visto na Tabela 4, todos os indicadores são satisfatórios, não havendo necessidade de outros ajustes.

Finalizando essa fase da análise, observamos outros dois indicadores. O primeiro, denominado indicador de Stone-Geisser da validade preditiva $\left(\mathrm{Q}^{2}\right)$, avalia quanto o modelo se aproxima do que se esperava dele ou, em outras palavras, a acurácia do modelo ajustado (Ringle, Silva, \& Bido, 2014). Já o segundo, denominado indicador de Cohen do tamanho 
do efeito ( $\mathrm{f}^{2}$ ), é obtido pela inclusão e exclusão de constructos do modelo (um a um) e avalia o quanto cada construto é útil para o ajuste do modelo estimado (Hair, Hult, Ringle, \& Sarstedt, 2016; Ringle, Silva, \& Bido, 2014).

O resultado dessa análise final é apresentado na Tabela 6, pela qual é possível admitir que não há problemas de estimação do modelo proposto.

Tabela 6

Validade preditiva e tamanho do efeito do modelo

\begin{tabular}{|c|c|c|}
\hline Construtos & CV RED $\left(Q^{2}\right)$ & CV COM $\left(f^{2}\right)$ \\
\hline Facilidade de uso percebida & 0,332 & 0,393 \\
\hline Intenção de uso & 0,427 & 0,539 \\
\hline Percepção do número de usuários & 0,138 & 0,483 \\
\hline Utilidade Percebida & 0,226 & 0,372 \\
\hline Valor da tecnologia & 0,285 & 0,378 \\
\hline $\begin{array}{l}\text { Valores críticos segundo Hair et al. } \\
\text { (2016) e Ringle, Silva e Bido } \\
\text { (2014) }\end{array}$ & $\mathrm{Q}^{2}>\mathrm{O}$ & $\begin{array}{c}\text { o,o2, o,15 e o,35 são } \\
\text { considerados pequenos, } \\
\text { médios e grandes } \\
\text { respectivamente }\end{array}$ \\
\hline
\end{tabular}

Fonte: dados da pesquisa (2018)

Passamos, então, à análise dos caminhos estruturais do modelo.

\section{Análise Dos Caminhos}

A modelagem de equações estruturais (MEE) e a respectiva análise de caminhos foram realizadas após a validação convergente e discriminante. O resultado encontrado, conforme retratado na Figura 4, indica que a relação entre todos os construtos foi positiva. 


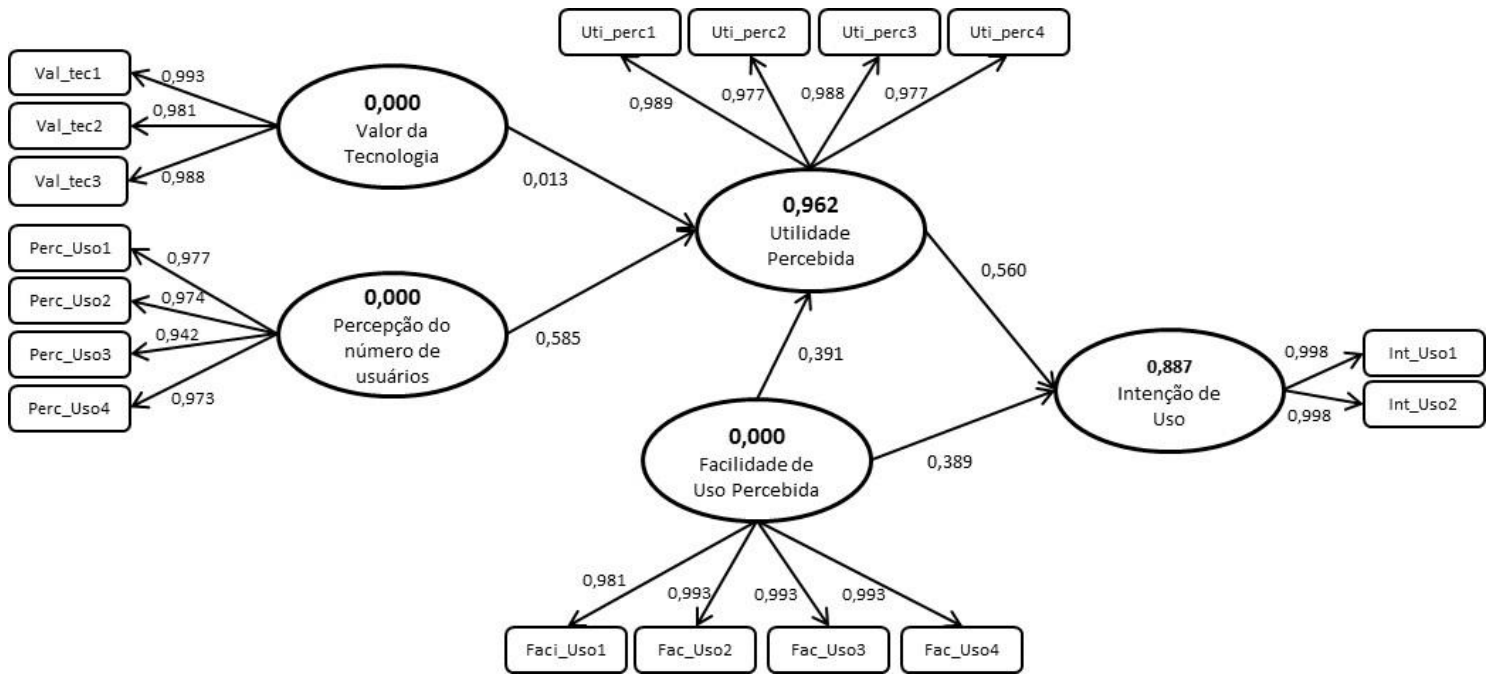

Figura 4. Modelo TAM estendido adaptado à realidade brasileira

Fonte: elaborada pelos autores

Porém, como amplamente recomendado por estudiosos de MEEs conduzidas por meio dos mínimos quadrados parciais, é necessário que se verifique a significância das cargas dos caminhos, por meio de um processo de reamostragem. Como o software utilizado para a análise da MEE, neste estudo, foi o SmartPLS, optou-se pelo procedimento de reamostragem por bootstrapping (Chin, 1998).

Nesse procedimento, os coeficientes dos caminhos são comparados com as médias de repetições com substituição da amostra original (Ringle, Silva, \& Bido, 2014). Essa verificação é realizada por meio do teste $\mathrm{t}$ (Student). Caso o resultado seja grande o suficiente, infere-se que o teste é significativo, pois a diferença encontrada é verdadeira e não decorrente da variabilidade da amostra. Quando não, assume-se que o caminho é não significativo.

A estimação do teste $t$ foi realizada por meio de 200 repetições dos 147 casos, em que foram avaliadas a significância estatística da diferença entre duas médias de amostras independentes.

As hipóteses propostas para modelo de aceitação do MB no Brasil foram testadas e analisadas. Somente o caminho entre o Valor da Tecnologia e a Utilidade Percebida não apresentou significância estatística e precisou ser rejeitado. Os demais resultados do teste de caminhos são apresentados na Tabela 7.

Tabela 7

Análise do modelo estrutural 


\begin{tabular}{|c|c|c|c|c|c|c|}
\hline Hipóteses & Caminho & $\begin{array}{l}\text { Carga } \\
\text { original }\end{array}$ & $\begin{array}{c}\text { Média das } \\
200 \\
\text { repetições } \\
\text { em } \\
\text { bootstrap }\end{array}$ & $\begin{array}{c}\text { Erro } \\
\text { padronizado }\end{array}$ & $\begin{array}{c}\text { Teste } \\
t\end{array}$ & $\begin{array}{c}p- \\
\text { value }\end{array}$ \\
\hline $\mathrm{H} 1$ & Valor da tecnologia -> Utilidade Percebida & 0,013 & $-0,007$ & 0,221 & 0,058 & n.s. \\
\hline $\mathrm{H} 2$ & $\begin{array}{l}\text { Percepção do número de usuários -> } \\
\text { Utilidade Percebida }\end{array}$ & 0,585 & 0,614 & 0,285 & 2,053 & $\mathrm{p}<0,05$ \\
\hline $\mathrm{H}_{3}$ & Utilidade Percebida -> Intenção de uso & 0,560 & 0,564 & 0,113 & 4,954 & $\mathrm{p}<\mathrm{O}, \mathrm{O} 1$ \\
\hline $\mathrm{H}_{4} \mathrm{a}$ & $\begin{array}{l}\text { Facilidade de uso percebida -> Utilidade } \\
\text { Percebida }\end{array}$ & 0,391 & 0,382 & 0,091 & 4,295 & $\mathrm{p}<\mathrm{O}, \mathrm{O} 1$ \\
\hline $\mathrm{H} 4 \mathrm{~b}$ & $\begin{array}{l}\text { Facilidade de uso percebida -> Intenção de } \\
\text { uso }\end{array}$ & 0,608 & 0,601 & 0,080 & 7,565 & $\mathrm{p}<\mathrm{O}, \mathrm{O} 1$ \\
\hline
\end{tabular}

Fonte: dados da pesquisa (2018)

\section{Discussão Dos Resultados}

O estudo feito por Wang, Fang e Lo (2008) trouxe o Valor da Tecnologia e o Número de Usuários como variáveis significantes no processo de adoção de um novo bem ou serviço no setor das telecomunicações, além das variáveis Utilidade Percebida e Facilidade de Uso Percebida, que já são contempladas desde a formulação original do Modelo TAM (Davis, 1989). Essas novas variáveis foram acrescentadas ao modelo, criando um TAM estendido, adaptado às inovações que possuem externalidades de rede.

O teste conduzido neste estudo encontrou resultados divergentes do modelo proposto e testado anteriormente (Wang, Fang, \& Lo, 2008). Apesar da relação entre a percepção do número de usuários e a utilidade percebida ter se mostrado positiva e significante, o valor da tecnologia não respondeu da mesma maneira, apresentando uma carga não-significativa de influência na percepção de utilidade.

$\mathrm{O}$ resultado encontrado pode ser justificado pelo fato de que os usuários de MB analisados ainda não tem a percepção de tecnologia muito bem acurada, já que muitas vezes acreditam não possuir os bens mais modernos disponíveis. No survey aplicado, a assertiva que discutia se a maioria dos smartphones no Brasil estava preparada para receber as tecnologias oferecidas pelo $\mathrm{MB}$ apresentou um score geral mediano $(\mu=4)$, sendo um dos mais baixos de toda a pesquisa. Com isso, concluímos que, apesar do número de smartphones estar crescendo a níveis exponenciais (Bessa, 2014), ainda há um longo caminho a percorrer até que o valor agregado da tecnologia consiga ser percebido pelo consumidor e se torne uma variável relevante no processo de tomada de decisão, assim como ocorre em outros países. 
Porém, mesmo o valor da tecnologia possuindo considerável significância, quando testado em outros países (Wang, Fang, \& Lo, 2008), a variável percepção do número de usuários é a responsável pela maior assimilação de ganho de benefício percebido.

O teste da $\mathrm{H} 2$ obteve resultados que seguiam a tendência esperada. A percepção do número de usuários apresentou uma carga elevada $(\Gamma=0,585)$, se mostrando a variável de maior influência na definição da utilidade percebida. Esse resultado pode estar ligado ao fato da população brasileira possuir um fator "social" muito mais relevante no seu cotidiano, quando comparada a outras sociedades. O resultado obtido pode ser explicado pelo conceito de que o brasileiro é uma espécie de "homem cordial", em que o aspecto social é a parcela que mais importa na sua vida em sociedade (Holanda, 1995; Brito, 2018).

Outro aspecto que contribui para o alto valor, dada a variável percepção do número de usuários, é o engajamento dos brasileiros nas redes sociais. De acordo com o relatório que discute o impacto das mídias sociais, publicado pela Kantar (2016), os brasileiros compõem a nacionalidade que mais interage em mídias sociais, um indicativo de que as externalidades de redes no setor de comunicações teriam um efeito multiplicador bastante considerável, se levado em consideração o benefício percebido pelo número de adotantes. Além disso, brasileiros compartilham um padrão de orientação mais tendente do coletivismo (Bond \& Smith, 1993; Clemente \& Gouveia, 2000).

As hipóteses destinadas a confirmar a influência da Utilidade Percebida na Intenção de Uso (H3), da Facilidade Percebida na Utilidade Percebida (H4a), e da Facilidade de Uso Percebida na Intenção de Uso ( $\left.\mathrm{H}_{4} \mathrm{~b}\right)$ foram suportadas com cargas positivas significantes, assim como já havia sido verificado em estudos anteriores que traziam o modelo TAM como base teórica e empírica (Davis; 1989; Wang, Fang, \& Lo, 2008).

No Brasil, o processo de adoção do serviço de MB está intimamente ligado à percepção do número de usuários que utilizam o serviço, sendo essa variável endossada pelo "perfil social” do brasileiro.

\section{Principais Contribuições deste Estudo}

A principal contribuição deste estudo, alinhado com seu objetivo, foi identificar o efeito da percepção do número de usuários e do valor da nova tecnologia como antecedente do modelo TAM na estimação da intenção de uso da referida tecnologia. Os resultados indicam que o valor percebido da tecnologia não influencia a intenção de uso ao mesmo 
tempo em que a percepção do número de usuários tem efeito positivo e significante na intenção comportamental.

Outra contribuição deste estudo é apontar para os desenvolvedores de tecnologia e gestores das grandes corporações da área das telecomunicações atuantes no Brasil que a aceitação de tecnologias no país responde de maneira peculiar. O elemento com maior poder de influência na percepção de utilidade é o número de usuários, ou seja, se torna extremamente importante fazer um bom trabalho de difusão e divulgação dos novos bens e serviços que possuem externalidades de rede, para que, quando atingido o grande público, os consumidores tenham a percepção de que a adoção é grande e largamente aceita.

O benefício adquirido por meio da percepção do número de usuários é, no Brasil, fator chave do sucesso de qualquer bem ou serviço novo no mercado. É essencial que as companhias estejam cientes da importância de terem seus produtos totalmente inseridos nesse cenário de externalidade de rede.

Os resultados indicaram ainda que a intenção de uso de uma nova tecnologia é - em grande medida - explicada pela Utilidade Percebida e pela Facilidade de Uso percebida pelos consumidores. Com isso, uma das contribuições deste estudo é a constatação de que o modelo concebido por Davis (1986) e adaptado por Wang, Fang e Lo (2008) ainda é eficiente para estimação da intenção de uso de novas tecnologias.

Assim como visto em estudos anteriores que analisaram consumidores finais (Goodman, Cryder, \& Cheema, 2013; Peterson \& Merunka, 2014; Bhatti, 2015; Chintalapati \& Daruri, 2017, Islam, \& Buxmann, 2018), o efeito da Facilidade Percebida na intenção de uso é maior do que o efeito da Utilidade percebida.

Sendo assim, tendo claro quais são as variáveis comportamentais e os fatores que definem e influenciam a aceitação tecnológica no setor das telecomunicações no Brasil, ficam identificadas quais as principais diferenciações do país em relação ao comportamento do consumidor no resto do mundo. E assim, é possível traçar planos estratégicos mais eficientes e ferramentas mais precisas para os pesquisadores da área no contexto nacional.

\section{Limites do Trabalho e Considerações Para Pesquisas Futuras}

O comportamento do consumidor é um tema bastante complexo e possui diferentes vertentes de abordagem. O modelo TAM estendido, conforme trazido por este estudo, 
testou o efeito das variáveis advindas das externalidades de rede no setor das telecomunicações, mas não esgotou todas as possíveis variáveis que possam influenciar o processo de tomada de decisão e adoção de uma nova tecnologia. Portanto, uma possível continuação deste trabalho é identificar, avaliar e testar quais seriam outras possíveis externalidades que também interferem na aceitação de um bem ou serviço.

Também, pode-se considerar que a amostra utilizada é pouco representativa, devido ao seu número limitado e pouco diverso. O público analisado foi muito concentrado em um mesmo perfil, com características específicas. Sugere-se que estudos futuros analisem uma amostra maior e com diferentes perfis, dessa forma, obter-se-á uma generalização mais assertiva.

Além disso, também seria um ganho à comunidade acadêmica, caso a análise dos dados fosse realizada em corte temporal transversal, para verificar-se a evolução da adoção desse serviço.

Nota: os autores agradecem pelas excelentes sugestões de melhoria recebidas dos pareceristas anônimos da TPA nas fases iniciais do processo de revisão. 


\section{Referências}

Anderson Jr, E. G., Parker, G. G., \& Tan, B. (2013). Platform performance investment in the presence of network externalities. Information Systems Research, 25(1), 152-172.

Almasri, A. K. M. (2018). New Mobile Learning Process Model for Higher Education Students in Jordanian Universities. International Journal of Information, Business and Management, 10(1), 201-213.

Bagozzi, R., \& Philips, L. (1982). Assessing construct validity in organizational research. Administrative Science Quartely, 36(1), 421-458.

Battistella, C., Colucci, K., De Toni, A. F., \& Nonino, F. (2013). Methodology of business ecosystems network analysis: A case study in Telecom Italia Future Centre. Technological Forecasting and Social Change, 8o(6), 1194-1210.

Bessa, I. (2014). Relatório Google sobre o uso de smartphones no Brasil. 2012. Recuperado de http://www. slideshare. net/ivilabessa/relatrio-google-sobre-uso-desmartphone-no-brasil-maio2012.

Bhatti, T. (2015). Exploring factors influencing the adoption of mobile commerce. The Journal of Internet Banking and Commerce, 2007.

Bond, M.H., \& Smith, P.B.(1996). Cross-cultural social and organizational psychology. Annual Review of Psychology, 47(1), 205-235. Recuperado de http://dx.doi.org/10.1146/annurev.psych.47.1.205.

Brito, J. G. (2018). Estado e desenvolvimento no pensamento de Octavio Ianni. Perspectivas: Revista de Ciências Sociais, 48.

Chih-Chien, W., Hsu, Y., \& Fang, W. (2005). Acceptance of technology with network externalities: an empirical study of internet instant messaging services. JITTA: Journal of Information Technology Theory and Application, 6(4), 15-25.

Chin, W. W. (1998). Commentary: Issues and opinion on structural equation modeling. Recuperado de http://www.jstor.org/stable/249674?seq=1\#page_scan_tab_contents 
Chin, W. W., \& Newsted, P. R. (1999). Structural equation modeling analysis with small samples using partial least squares. Statistical strategies for small sample research, 1(1), 307-341.

Churchill, G. A., \& Iacobucci, D. (2006). Marketing research: methodological foundations. New York: Dryden Press.

Chintalapati, N., \& Daruri, V. S. K. (2017). Examining the use of YouTube as a learning resource in higher education: scale development and validation of TAM model. Telematics and Informatics, 34(6), 853-860.

Conner, K. R. (1995). Obtaining strategic advantage from being imitated: When can encouraging “clones” pay?. Management Science, 41(2), 209-225.

Davis, F. D. (1989). Perceived usefulness, perceived ease of use and user acepptance of information technology. MIS Quarterly, 13(3),319-340.

Davis, F. D.(1993). User acceptance of information technology: system characteristics, user perceptions and behavioral impacts. International Journal of Man-Machine Studies, 38(3), $475-487$.

Davis, F. D., \& Venkatesh, V. (1996), A critical assessment of potential measurement biases in the technology acceptance model: Three experiments. International Journal HumanComputer Studies, 45(1), 19-45.

Davis, F. D., Bagozzi, R. P., \& Warshaw, P. R. (1989), User Acceptance of Computer Technology: A Comparison of Two Theoretical Models. Management Science, 35(8), 982-1003.

Faul, F., Erdfelder, E., Buchner, A., \& Lang, A. (2009). Statistical power analyses using $\mathrm{G}^{*}$ Power 3.1: Tests for correlation and regression analyses. Behavior Research Methods, 41(4), 1149-1160

Fornell, C., \& Larcker, D. (1982). Evaluating structural equation models with unobservable variables and measurement error. Journal of Marketing Research, 17(1), 39-50

Furr, R. M. (2017). Psychometrics: an introduction. Sage Publications. 
Gandal, N. (1994). Hedonic price indexes for spreadsheets and an empirical test for network externalities. the RAND Journal of Economics, 160-170.

Gangwar, H., Date, H., \& Ramaswamy, R. (2015). Understanding determinants of cloud computing adoption using an integrated TAM-TOE model. Journal of Enterprise Information Management, 28(1), 107-130.

Goodman, J. K., Cryder, C. E., \& Cheema, A. (2013). Data collection in a flat world: The strengths and weaknesses of Mechanical Turk samples. Journal of Behavioral Decision Making, 26(3), 213-224.

Gouveia, V.V., Clemente, M. (200o). O Individualismo-Coletivismo no Brasil e na Espanha: Correlatos Sócio-Demográficos. Estudos de Psicologia, 5 (2), 1-20.

Hair, J. F., Anderson, R. E., Tatham, R. L., \& Black, W. C. (2005). Análise multivariada de dados. Porto Alegre: Bookman.

Hair, J. F., Hult, G. T. M., Ringle, C., \& Sarstedt, M. (2016). A primer on partial least squares structural equation modeling (PLS-SEM). Sage Publications.

Hannan, T. H., \& McDowell, J. M. (1984). The determinants of technology adoption: The case of the banking firm. The RAND Journal of Economics, 328-335.

Holanda, S. B. D. (1995). Raízes do Brasil (1936). São Paulo: Companhia das Letras.

Hong, H., Cao, M., \& Wang, G. A. (2017). The effects of network externalities and herding on user satisfaction with mobile social APPs. Journal of Electronic Commerce Research, 18(1), 18.

Islam, N., \& Buxmann, P. (2018, January). The Role of Collaboration between Incumbent Firms and Start-ups on Customers' Adoption of Digital Innovation. In Proceedings of the 51st Hawaii International Conference on System Sciences.

Kantar. Social Media Impact Report 2016. China: Kantar, 2016. Color. Recuperado de http://www.cn.kantar.com/media/1202327/kantar_social_media_impact_report_mob ile_infographic.pdf 
Katz, M.L. \& Shapiro, C. (1986). Technology adoption in the presence of network externalities. Political Economy, 94(4), 822-841.

Kauffman, R. J., McAndrews, J., \& Wang, Y. M. (2000). Opening the "black box" of network externalities in network adoption. Information Systems Research, 11(1), 61-82.

Kupczik, V. (2009). Pesquisa exploratória sobre avaliação ergonômica de interfaces de sites de mobile banking brasileiras para iPhone (Dissertação de Mestrado). Universidade Federal do Paraná.

Malhotra, N. K. (2012). Pesquisa de marketing: uma orientação aplicada. Bookman Editora.

Masson, S., Jain, R., Ganesh, N. M., \& George, S. A. (2016). Operational efficiency and service delivery performance: a comparative analysis of Indian telecom service providers. Benchmarking: An International Journal, 23(4), 893-915.

Mohammadi, H. (2015). Investigating users' perspectives on e-learning: An integration of TAM and IS success model. Computers in Human Behavior, 45, 359-374.

Niu, Y. H., Lu, W. S., \& Liu, D. D. (2018). RFID-Enabled Management System Adoption and Use in Construction: Passing Through the Labyrinth with an Improved Technology Acceptance Model. In Proceedings of the 21st International Symposium on Advancement of Construction Management and Real Estate (pp. 1251-1258). Springer, Singapore.

Pando-Garcia, J., Periañez-Cañadillas, I., \& Charterina, J. (2016). Business simulation games with and without supervision: An analysis based on the TAM model. Journal of Business Research, 69(5), 1731-1736.

Peterson, R. A. (2001). On the use of college students in social science research: Insights from a second-order meta-analysis. Journal of consumer research, 28(3), 450-461.

Peterson, R. A., \& Merunka, D. R. (2014). Convenience samples of college students and research reproducibility. Journal of Business Research, 67(5), 1035-1041.

Rachman, T., \& Napitupulu, D. (2018). User acceptance analysis of potato expert system application based on TAM approach. Int. J. Adv. Sci. Eng. Inf. Technol, 8(1), 185-191. 
Ramos, A. S. M., Pimenta, I. L., \& Rodrigues, P. A. B. (2010). Diferenças de percepção de adotantes e não-adotantes quanto ao uso de serviços de mobile banking e sua relação com as características individuais de inovatividade. Revista pensamento contemporâneo em administração, 4(3), 34-43.

Rauniar, R., Rawski, G., Yang, J., \& Johnson, B. (2014). Technology acceptance model (TAM) and social media usage: an empirical study on Facebook. Journal of Enterprise Information Management, 27(1), 6-30.

Püschel, J., Afonso Mazzon, J., \& Mauro C. Hernandez, J. (2010). Mobile banking: Proposition of an integrated adoption intention framework. International Journal of Bank Marketing, 28(5), 389-409.

Santos, D. O., Veiga, R. T., \& Moura, L. R. C. (2011). Teoria do Comportamento Planejado Decomposto: determinantes de utilização do serviço mobile banking. Revista Organizações em Contexto-online, 6(12), 78-106.

Sánchez-Prieto, J. C., Olmos-Migueláñez, S., \& García-Peñalvo, F. J. (2017). MLearning and pre-service teachers: An assessment of the behavioral intention using an expanded TAM model. Computers in Human Behavior, 72, 644-654.

Silva, L. F., Schroder, T. A., \& Kroenke, A. (2018). Motivações e resistências para a utilização do mobile banking. Revista Empreender e Inovar, 1(1), 88-103.

Torrent-Sellens, J. (2015). Knowledge products and network externalities: implications for the business strategy. Journal of the Knowledge Economy, 6(1), 138-156.

Venkatesh, V., \& Davis, F. D. (2000). A theoretical extension of the technology acceptance model: Four longitudinal field studies. Management science, 46(2), 186-204.

Venkatesh, V., Morris, M. G., Davis, G. B., \& Davis, F. D. (2003). User acceptance of information technology: Toward a unified view. MIS quarterly, 425-478.

Wang, C. C., Lo, S. K., \& Fang, W. (2008). Extending the technology acceptance model to mobile telecommunication innovation: The existence of network externalities. Journal of consumer Behaviour, 7(2), 101-110. 
Wua, J.H., \& Wang, S. C. (2002). An empirical study of consumers adopting mobile commerce in Taiwan: imalyzed by structural equation modeling. Proceedings of the 7 th Pacific. Asia Conference on hifonnation Systems, Australia

Zarifopoulos, M., \& Economides, A. A. (2008). Evaluating mobile banking portals. International Journal of Mobile Communications, 7(1), 66-90.

Zhou, T. (2018). Examining users' switch from online banking to mobile banking. International Journal of Networking and Virtual Organisations, 18(1), 51-66.

Zott, C., \& Amit, R. (2010). Business model design: an activity system perspective. Long range planning, 43(2), 216-226. 